\title{
Wound Healing Study of Eucalyptus Essential Oil Containing Nanoemulsion in Rat Model
}

\author{
Prawez Alam ${ }^{1}$, Faiyaz Shakeel ${ }^{2 *}$, Md Khalid Anwer ${ }^{3}$, Ahmed I. Foudah ${ }^{1}$ and \\ Mohammed H. Alqarni ${ }^{1}$ \\ ${ }^{1}$ Department of Pharmacognosy, College of Pharmacy, Prince Sattam Bin Abdulaziz University, P.O. Box 173, AI-Kharj 11942, SAUDI ARABIA
2 Kayyali Chair for Pharmaceutical Industries, Department of Pharmaceutics, College of Pharmacy, King Saud University, P.O. Box 2457,
Riyadh 11451, SAUDI ARABIA
${ }^{3}$ Department of Pharmaceutics, College of Pharmacy, Prince Sattam Bin Abdulaziz University, P.O. Box 173, AI-Kharj 11942, SAUDI ARABIA
}

\begin{abstract}
The objective of this investigation was to develop nanoemulsion formulations of Eucalyptus essential oil (EEO) and to evaluate its wound healing effects in comparison with standard gentamycin in rat model. Various nanoemulsionns of EEO were prepared using aqueous phase titration method and the zones of nanoemulsion were identified by the construction of phase diagrams. EEO nanoemulsions were investigated in terms of physical stability, self-nanoemulsification efficiency and physicochemical characterization. Optimized nanoemulsion of EEO was selected for wound healing study, collagen estimation and histopathological evaluation in rats in comparison with pure EEO and standard gentamycin. Optimized nanoemulsion presented significant would healing activity in rats as compared with pure EEO upon oral administration. The wound healing activity of optimized nanoemulsion was comparable with standard gentamycin. Optimized EEO nanoemulsion also presented significant enhancement in collagen content as compared with pure EEO and negative control. However, the collagen contents of optimized nanoemulsion treated animals were comparable with standard gentamycin-treated animals. Histopathological studies of optimized nanoemulsion treated rats showed no signs of inflammatory cells which suggested the safety and non-toxicity of EEO nanoemulsion. This study suggested the potential of nanoemulsion in enhancing the wound healing activity of EEO upon oral administration.
\end{abstract}

Key words: collagen estimation, Eucalyptus oil, nanoemulsion, wound healing

\section{INTRODUCTION}

Essential oils got great acceptance in pharmaceutical and food industries due to their broad range of therapeutic activities and flavoring effects ${ }^{1-4)}$. The main chemicals of essential oils are terpenes which are responsible for most of their therapeutic activity ${ }^{4)}$. Eucalyptus essential oil (EEO) is isolated and extracted from the dried leaves of Eucalyptus citriodora and other species of Eucalyptus ${ }^{5,6)}$. Various therapeutic activities such as analgesic, anti-inflammatory $^{5)}$, antioxidant ${ }^{7)}$, antifungal ${ }^{8)}$, antibacterial ${ }^{6,9)}$ and antiradical activities ${ }^{7}$ have been reported for EEO in literature. Various terpenes such as $\alpha$-pinene, limonene, 1,8-cineole, p-cymene, tr-pinocarveol, $\alpha$-terpineol, globulol, $\alpha$-eudesmol, $\beta$-eudesmol and others have been detected in different species of $\mathrm{EEO}^{6,8,9)}$. The main component/biomarker responsible for the most of the therapeutic activities of EEO is 1,8-cineole (cineole or eucalyptol) ${ }^{6,10}$.
Oral nanoemulsions have got great acceptance as drug delivery carriers for the enhancement of therapeutic activities of various therapeutic agents and bioactive compounds in recent years ${ }^{11-14)}$. Nanoemulsions offer several advantages over other colloidal drug carriers due to their ease of preparation, low preparation cost, physical/thermodynamic stability and nanosized droplet diameters ${ }^{14-16)}$. In the recent years, would healing power of several essential oils have been proposed in various animal models ${ }^{17-26)}$. However, the wound healing activities of most of the essential oils using nanosized drug delivery systems have rarely been studied in literature. The wound healing activity of clove essential oil (CEO) via nanoemulsion has been reported recently in literature ${ }^{12)}$. Alam et al. got significant wound healing effects of CEO loaded nanoemulsion as compared with pure $\mathrm{CEO}^{12)}$. The wound healing effects of EEO topical nanoemulsion have also been reported in liter-

\footnotetext{
*Correspondence to: Faiyaz Shakeel, Kayyali Chair for Pharmaceutical Industries, Department of Pharmaceutics, College of Pharmacy, King Saud University, P.O. Box 2457, Riyadh 11451, SAUDI ARABIA

E-mail: faiyazs@fastmail.fm

Accepted March 10, 2018 (received for review January 14, 2018)

Journal of Oleo Science ISSN 1345-8957 print / ISSN 1347-3352 online

http://www.jstage.jst.go.jp/browse/jos/ http://mc.manusriptcentral.com/jjocs
} 
$\operatorname{ature}^{27)}$. However, the wound healing effects of EEO nanoemulsion upon oral administration have not been studied in literature. Hence, the objective of this research work was to develop suitable nanoemulsion formulations of EEO and to investigate its wound healing effects in rat model as compared with pure EEO and standard antibiotic (gentamycin)after oral administration. Macroemulsions were not prepared in this work because nanoemulsions have lower droplet size in comparison with macroemulsions and these systems are more suitable for enhancing absorption and therapeutic activity of essential oils ${ }^{11,12,27)}$. Various nanoemulsion formulations of EEO were prepared using aqueous phase titration method via construction of pseudoternary phase diagrams. The components of nanoemulsions were safe and nontoxic and includes EEO/oil phase, Tween-85/surfactant, Transcutol/cosurfactant and water/ aqueous phase. All the formulations were developed properly, characterized physicochemically and optimized nanoemulsion of EEO was taken for biological wound healing activity in rats.

\section{EXPERIMENTAL}

\subsection{Materials}

EEO, dimethyl sulfoxide (DMSO) and ethanol were obtained from "Sigma Aldrich (St. Louis, MO)". Diethylene glycol monoethyl ether (Transcutol-HP) was obtained from “Gattefossé(Lyon, France)". Polyoxyethylenesorbitan trioleate was obtained from "E-Merck(Darmstadt, Germany)". Ultra-pure water (deionized water) was obtained from "ELGA water purification system" in the laboratory. All other chemicals and reagents used were of analytical/pharmaceutical grades which were used without any further purification.

\subsection{Construction of pseudo-ternary phase diagrams for EEO nanoemulsion}

In order to develop various nanoemulsion formulations of EEO, EEO/oil phase, Tween-85/surfactant, Transcutol/ cosurfactant and deionized water/aqueous phase were used in this study. Pseudo-ternary phase diagrams were developed using aqueous phase titration method ${ }^{28,29)}$. In brief, Tween-85 and Transcutol were mixed thoroughly in different mass ratios (1:0, 1:2, 1:1, 2:1 and 3:1). EEO/oil phase and a particular mass ratio of surfactant to cosurfac$\operatorname{tant}\left(\mathrm{S}_{\text {mix }}\right)$ were then mixed at various mass ratios $(1: 9$ to 9:1). Pseudo-ternary phase diagrams constructed developed using aqueous phase titration method ${ }^{28)}$. In this method, the mixture of EEO and specific $\mathrm{S}_{\text {mix }}$ was titrated with drop-by-drop addition of aqueous phase and recorded for visual observations based on their clarity ${ }^{28,30)}$. The clear/transparent and easily flowable zones of nanoemulsion plotted constructed on respective pseudo-ternary phase diagram in which one axis representing the water, second EEO and third one representing the specific $\mathrm{S}_{\text {mix }}$.

\subsection{Formulation development of EEO}

It was observed from pseudo-ternary phase diagrams that the maximum nanoemulsion zones were shown by $1: 1$ $\mathrm{S}_{\text {mix }}$ ratio and hence the mass ratio of $1: 1$ was selected for the development of nanoemulsion formulations of EEO. Various nanoemulsions of EEO(E1-E5)were selected from the phase diagram of 1:1 mass ratio. The entire zones of nanoemulsion were considered in formulation selection. In formulations E1-E5, the concentration of EEO was varied from $12-28 \% \mathrm{w} / \mathrm{w}$ and the concentration of $\mathrm{S}_{\text {mix }}$ was kept constant at $24 \% \mathrm{w} / \mathrm{w}$. The formulation composition of EEO loaded nanoemulsions is furnished in Table 1. Typical photographs of various EEO nanoemulsions (E1-E5) are presented in Fig. 1 which indicated clear/transparent nature of prepared nanoemulsions.

\subsection{Physical stability tests}

Because in aqueous phase titrations, observations were made visually, there is possibility of formation of metastable or unstable formulation. Therefore, physical stability tests on developed EEO loaded nanoemulsions (E1-E5) were conducted in order to eliminate any unstable/metastable nanoemulsion. These tests were carried at various stress conditions including "centrifugation, heating \& cooling cycles and freeze-pump-thaw cycles” ${ }^{16,30)}$. EEO nanoemulsions (E1-E5) were centrifuged at $5000 \mathrm{rpm}$ for $30 \mathrm{~min}$ and observed for any physical change such as phase separation,

Table 1 Composition of nanoemulsion formulations of EEO (E1-E5) prepared using EEO, Tween-85, Transcutol and water.

\begin{tabular}{cccccc}
\hline \multirow{2}{*}{ Code } & \multicolumn{4}{c}{ Formulation composition $(\% \mathrm{w} / \mathrm{w})$} & \multirow{2}{*}{$\mathrm{S}_{\text {mix }}$ ratio } \\
\cline { 2 - 5 } & EEO & Tween-85 & Transcutol & Water & \\
\hline E1 & 12 & 12 & 12 & 64 & $1: 1$ \\
E2 & 16 & 12 & 12 & 60 & $1: 1$ \\
E3 & 20 & 12 & 12 & 56 & $1: 1$ \\
E4 & 24 & 12 & 12 & 52 & $1: 1$ \\
E5 & 28 & 12 & 12 & 48 & $1: 1$ \\
\hline
\end{tabular}




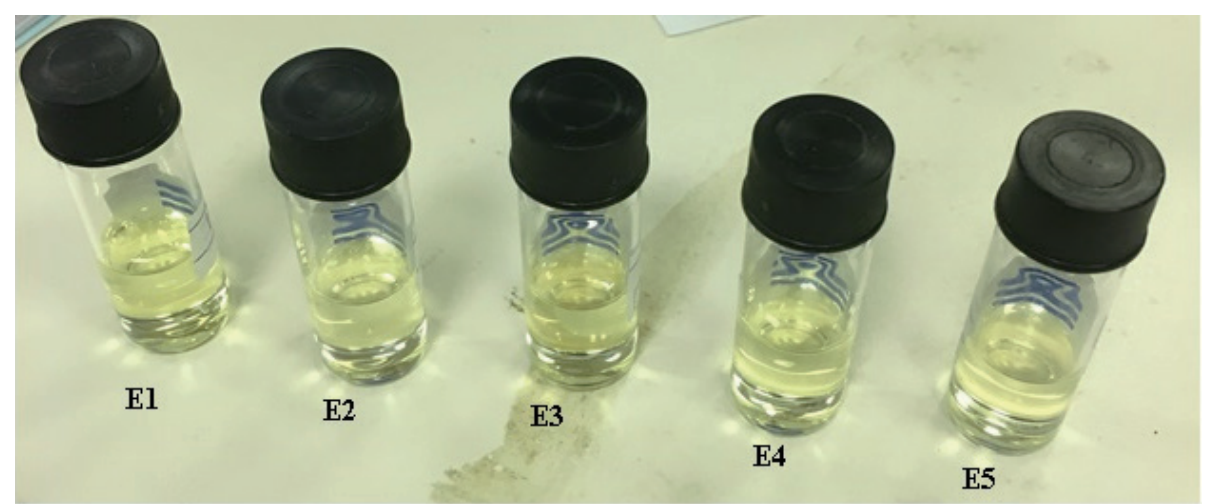

Fig. 1 Typical photographs of various EEO nanoemulsions (E1-E5) prepared using EEO (oil phase), water (aqueous phase), Twenn-85 (surfactant) and Transcutol(cosurfactant).

formation of macroemulsion, cracking or coalescence etc. Formulations which were stable at centrifugation were subjected to heating \& cooling cycles. Three heating \& cooling cycles were performed between $50^{\circ} \mathrm{C}$ and $4^{\circ} \mathrm{C}$ for the period of $48 \mathrm{~h}$ for each cycle and observed again for phase separation, formation of macroemulsion, cracking or coalescence etc. Formulations which were stable at heating \& cooling cycles were subjected to freeze-pump-thaw cycles. Three freeze-pump-thaw cycles were performed between $-21^{\circ} \mathrm{C}$ and $25^{\circ} \mathrm{C}$ for the period of $24 \mathrm{~h}$ for each cycle and observed again for phase separation, formation of macroemulsion, cracking or coalescence etc. Overall, formulations those were found to be stable at all three steps of physical stability tests were selected for further studies.

\subsection{Self-nanoemulsification efficiency tests}

The aim of "self-nanoemulsification test" was to assess any precipitation/phase separation upon mild agitation/dilution with three different diluents including "water, acid buffer $(0.1 \mathrm{~N} \mathrm{HCl})$ and phosphate buffer $(\mathrm{pH} 7.4)$ ”. This test was conducted by diluting $1.0 \mathrm{~mL}$ of each nanoemulsion (E1-E5) with "water, $0.1 \mathrm{~N} \mathrm{HCl}$ and phosphate buffer $(\mathrm{pH}$ 7.4)" . The dilution ratio of each nanoemulsion to particular diluent was kept constant at 1:500. The self-nanoemulsification power of each nanoemulsion was evaluated visually using A-E grading systems as described below ${ }^{16,30)}$ :

Grade A: Rapidly forming clear/transparent nanoemulsion

Grade B: Rapidly forming bluish slightly less clear nanoemulsion

Grade C: Slowly forming milky/turbid emulsions

Grade D: Dull, grayish slowly forming milky/turbid emulsions

Grade E: Milky/turbid emulsions with the presence of oil globules at the surface

\subsection{Physicochemical evaluation of EEO nanoemulsions}

Developed nanoemulsions of EEO were evaluated for various physicochemical parameters including "droplet diameter, polydispersity index (PDI), zeta potential(ZP), refractive index $(\mathrm{RI})$, the percentage of transmittance (\% T) and surface morphology using transmission electron microscopy (TEM)". The average droplet diameter, PDI and ZP of prepared nanoemulsions (E1-E5) were measured using "Malvern Particle Size Analyzer (Holtsville, NY)" at $25.0 \pm 1.0^{\circ} \mathrm{C}$. The scattering angle for this analysis was set at $90^{\circ}$. The detailed procedure for these measurements is given in our publications ${ }^{16)}$. The RIs of EEO nanoemulsions (E1-E5) were measured using "Abbes type Refractometer (Precision Testing Instruments Laboratory, Germany)" at $25 \pm 1{ }^{\circ} \mathrm{C}$ as proposed in literature ${ }^{16)}$. The \% T of EEO nanoemulsions (E1-E5) was measured spectrophotometrically at the wavelength of $550 \mathrm{~nm}$ as proposed in literature ${ }^{16)}$.

The surface morphology and droplets diameter of optimized EEO nanoemulsion(E1) was studied using "JEOL TEM technique (JEOL JEM-2100 F, USA)”. TEM analysis was performed under light microscopy which was operated at $100 \mathrm{KV}$. The detailed descriptions for TEM analysis are given in our previous publication ${ }^{29)}$.

\subsection{Wound healing evaluation in rat model}

Based on various physicochemical parameters such as minimum droplet diameter (32.45 nm), least PDI(0.153), optimal $\mathrm{ZP}$ value $(-34.25 \mathrm{mV})$, optimal $\mathrm{RI}$ value (1.335) and maximum \% T value $(99.1 \%)$, nanoemulsion formulation E1 was optimized and selected for wound healing activity in rats. For would healing study, female Albino Wistar rats (weighing from 200 to $250 \mathrm{~g}$ ) were taken from the "Experimental Animal Care Center at King Saud University, Riyadh, Saudi Arabia”. All the rats were provided controlled environmental conditions in terms of temperature and humidity with free access of water and standard pellet diet. This study on rats was approved by "Research Ethics Committee of College of Pharmacy, King Saud University, Riyadh, Saudi Arabia” and their guidelines were followed. Wound excision rat model was used for this purpose. The rats were divided randomly into four different groups con- 
taining six rats in each group. The rats were anaesthetized using diethyl ether and depilated at the predetermined site before wounding. An excision wound was produced by cutting away approximately $500 \mathrm{~mm}^{2}$ full thickness of the area on the anterior-dorsal side of each animal ${ }^{31)}$. Rats were treated by oral administration of various samples.

Group I(control group)rats were treated by oral administration of an optimized nanoemulsion E1 without EEO; group II animals were treated by oral administration of pure EEO (25 mg/kg of body weight); group III animals were treated by oral administration of an optimized nanoemulsion E1 with EEO (containing $25 \mathrm{mg} / \mathrm{kg}$ of EEO) and group IV animals were treated by oral administration of gentamycin suspension $(25 \mathrm{mg} / \mathrm{kg}$ ) which was taken as positive control group. Different formulations were administered orally to respective group once daily for the period of 24 days. Wound healing property of each group was evaluated in terms of the percent of wound contraction and closure time. The wound area was determined every third day $^{32}$. The time for wound closure was recorded when total wound healed.

\subsection{Collagen determination}

The skin pieces from the wound area of animals belong to each group were taken at the end of the experiment (after 24 days) and studied for the collagen content in each group. For collagen determination in skin, $5 \mathrm{~mL}$ of TES buffer and $0.1 \mathrm{~mL}$ of acid hydrosolate were taken in the tube, mixed well and incubated at $37^{\circ} \mathrm{C}$ for $6 \mathrm{~h}$. The contents were then filtered using a syringe filter into clean tube and allowed to stand. About $0.2 \mathrm{~mL}$ of test filterate was taken and $2 \mathrm{~mL}$ of ninhydrin reagent was added into filtrate and boiled for about $30 \mathrm{~min}$. After cooling at ambient temperature, $10 \mathrm{~mL}$ of 1-propanol was added and the absorbance was taken spectrophotometrically at the wavelength of $570 \mathrm{~nm}^{33,34)}$. The collagen content in terms of $\mathrm{mg} / \mathrm{g}$ of tissue was then calculated from the spectrophotometric absorbance. The skin pieces were also taken from untreated rats (control) and subjected for the analysis of leucine content. The leucine content in untreated rats was considered as negative control.

\subsection{Histopathology of tissues}

For histopathology studies, the healed tissues from the animals of each group were procured at the end of the experiment, fixed with $10 \%$ formalin, dehydrated using alcohol and embedded in paraffin blocks. Tissue sections were deparaffinized using xylene dye. Serial sections with specific diameter were cut using microtome and stained with hematoxylin-eosin (HE) dye. The tissue sections were studied using light microscopy. The severity of the inflammation in the healed areas was investigated by counting the inflammatory cell infiltration per field in each group. The presence of "epithelization, inflammatory cell infiltra- tion, fibroblast proliferation, neovascularization and collagen deposition" on healed area of skin were measured using a modified 0-5 numerical scale stated below ${ }^{12}$ :

Score 0: Absence

Score 1: Occasional presence

Score 2: Light scattering

Score 3: Abundance

Score 4: Confluence of cells

Score 5: Fibres

\subsection{Data analysis}

The values of in vitro evaluation/physicochemical investigation are presented as mean \pm standard deviation. However, the values of wound healing activity are presented as mean \pm standard error of mean (SEM). The statistical significance was analyzed using ANOVA in which $p<0.05$ was taken as statistically significant.

\section{RESULTS}

\subsection{Construction of pseudo-ternary phase diagrams and preparation of EEO nanoemulsions}

Pseudo-ternary phase diagrams were constructed using EEO, Tween-85, Transcutol and water for the development of EEO nanoemulsions. The results of phase diagram constructions are shown in Fig. 2. The results summary of aqueous phase titration is furnished in Table 2. From results recorded, it can be seen that $S_{\text {mix }}$ ratio of 1:0 (surfactant alone) presented very low nanoemulsion zones (Fig. 2a). The highest amount of EEO/oil phase that was found to be solubilized by this mass ratio was recorded as $13 \% \mathrm{w} /$ $\mathrm{w}$ using $62 \% \mathrm{w} / \mathrm{w}$ of $\mathrm{S}_{\text {mix }}$. When the amount of Transcutol/ cosurfactant was increased with respect to Tween-85/surfactant i.e. $S_{\text {mix }}$ ratio of 1:2, the nanoemulsion zones were found to be increased as compared to 1:0 ratio (Fig. 2b). The highest amount of EEO that was solubilized by $1: 2$ ratio was $22 \% \mathrm{w} / \mathrm{w}$ using $52 \% \mathrm{w} / \mathrm{w}$ of $\mathrm{S}_{\text {mix }}$. However, when the concentration of Tween-85 and Transcutol was kept equal i.e. $\mathrm{S}_{\text {mix }}$ ratio of $1: 1$, the nanoemulsion zones were increased significantly as compared with 1:0 and 1:2 $\mathrm{S}_{\text {mix }}$ ratios (Fig. 2c). The highest amount of EEO that was solubilized by this ratio was obtained as 36\% w/w using $55 \%$ $\mathrm{w} / \mathrm{w}$ of $\mathrm{S}_{\text {mix }}$ (Table 2). On the other hand, when the $\mathrm{S}_{\text {mix }}$ ratio of 2:1 was studied, the nanoemulsion zones were found to be decreased again in comparison with 1:1 $\mathrm{S}_{\text {mix }}$ ratio (Fig. 2d). The maximum concentration of EEO that was solubilized by this ratio was obtained as $28 \% \mathrm{w} / \mathrm{w}$ using $41 \% \mathrm{w} / \mathrm{w}$ of $\mathrm{S}_{\text {mix }}$ (Table 2). When the concentration of Tween-85 was increased further with respect to Transcutol i.e. $\mathrm{S}_{\text {mix }}$ ratio of 3:1, the nanoemulsion zones were found to be decreased again as compared to 1:1 and 2:1 ratios (Fig. 2e). The highest amount of EEO that was solubilized by this ratio was obtained as $24 \% \mathrm{w} / \mathrm{w}$ using $56 \% \mathrm{w} / \mathrm{w}$ of 

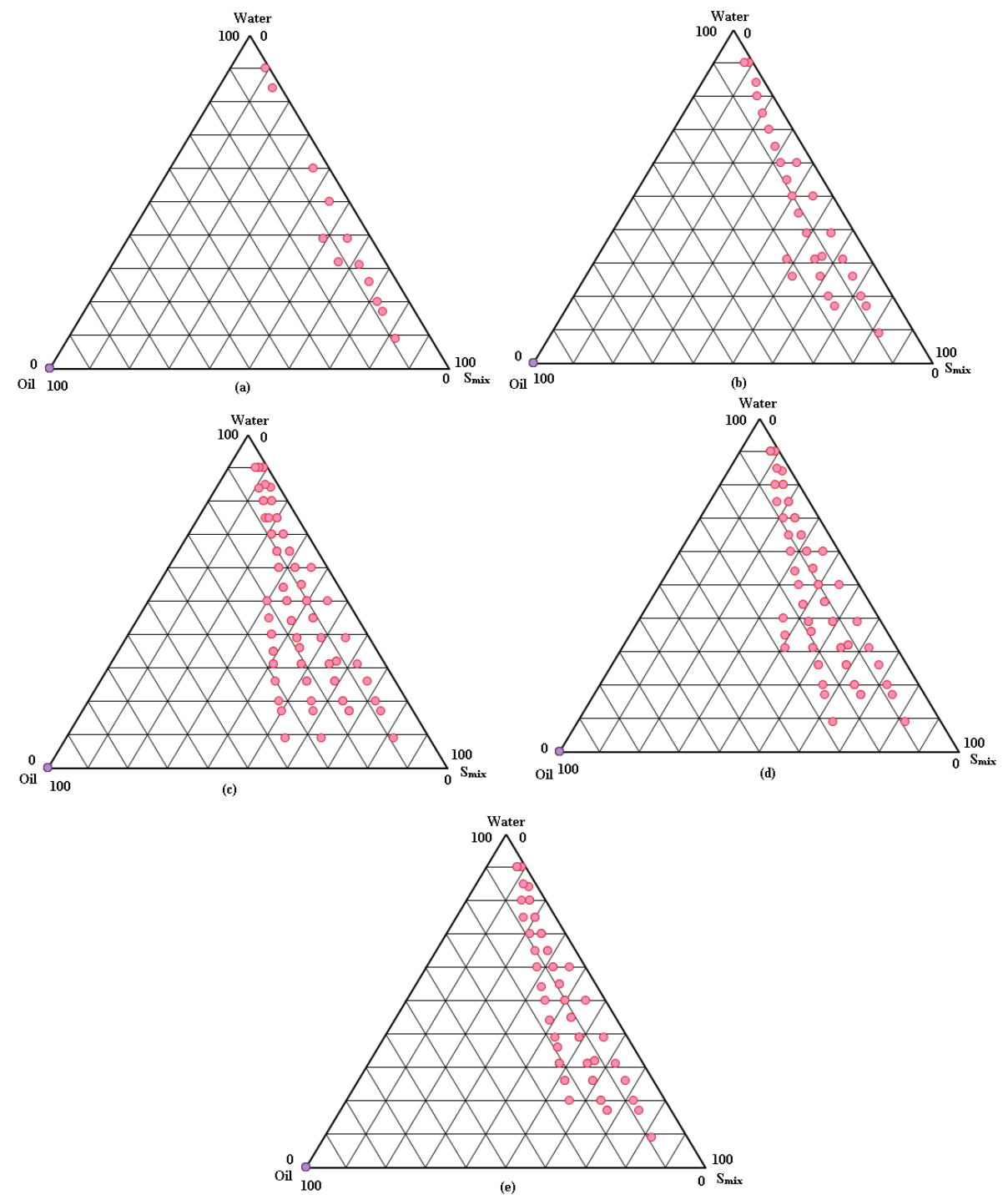

Fig. 2 Pseudo-ternary phase diagrams constructed via aqueous phase titration method for nanoemulsion zones (dotted area) for EEO (oil phase), water (aqueous phase), Twenn-85 (surfactant) and Transcutol (cosurfactant) at $\mathrm{S}_{\text {mix }}$ ratios of a. $1: 0$, b. $1: 2$, c. $1: 1, \mathrm{~d} 2: 1$ and e. $3: 1$.

Table 2 Results summary for aqueous phase titration of nanoemulsions.

\begin{tabular}{ccccccc}
\hline Figure & $\mathrm{S}_{\text {mix }}$ ratio & Surfactant & Cosurfactant & Nanoemulsion zones & $\begin{array}{c}\text { Oil phase solubilized } \\
(\% \mathrm{w} / \mathrm{w})^{\mathrm{a}}\end{array}$ & $\begin{array}{c}\mathrm{S}_{\text {mix }} \text { solubililized } \\
(\% \mathrm{w} / \mathrm{w})^{\mathrm{b}}\end{array}$ \\
\hline 1a & $1: 0$ & Tween-85 & Transcutol & Low & 13 & 62 \\
1b & $1: 2$ & Tween-85 & Transcutol & Higher than 1a & 22 & 52 \\
1c & $1: 1$ & Tween-85 & Transcutol & Highest & 36 & 55 \\
1d & $2: 1$ & Tween-85 & Transcutol & Lower than 1c & 28 & 41 \\
1e & $3: 1$ & Tween-85 & Transcutol & Lower than 1c \& 1d & 24 & 56 \\
\hline
\end{tabular}

${ }^{a}$ The maximum amount of EEO (oil phase) that was solubilized

${ }^{\mathrm{b}}$ The maximum amount of $\mathrm{S}_{\text {mix }}$ (Tween-85:Transcutol) phase that was solubilized with respect to maximum amount of oil phase 
$\mathrm{S}_{\text {mix }}($ Table 2).

\subsection{Physical stability tests}

The aim of physical stability tests was to eliminate any metastable or unstable nanoemulsions. Therefore, prepared EEO nanoemulsions were subjected to different physical stability tests. The qualitative results of physical stability tests are furnished in Table 3.

\subsection{Self-nanoemulsification tests}

Prepared nanoemulsions of EEO(E1-E5) were further evaluated for self-nanoemulsification test ${ }^{29)}$. The qualitative results of this test are also furnished in Table 3. It was observed that nanoemulsion formulations (E1-E3) passed this test with grade A and formulations E4 and E5 passed this test with grade $\mathrm{B}$ in the presence of all three diluents.

\subsection{Physicochemical evaluation of EEO nanoemulsions}

The results recorded for physicochemical evaluation of prepared EEO nanoemulsions (E1-E5) are furnished in Table 4. It was observed that the droplet diameter of EEO nanoemulsions (E1-E5) was obtained in the range of 32.45$142.35 \mathrm{~nm}$ (Table 4). The PDIs of EEO nanoemulsions (E1-E5) were obtained as 0.153-0.278 (Table 4). The least PDI was obtained in EEO nanoemulsion E1 (0.153), indi- cating the highest uniformity of droplet diameter as compared to other formulations.

The ZP values for nanoemulsions (E1-E5) were obtained as -38.25 to $-34.25 \mathrm{mV}$ (Table 4$)$. The least $\mathrm{ZP}$ value was obtained in nanoemulsion $\mathrm{E} 1(-38.25 \mathrm{mV})$. However, the maximum $\mathrm{ZP}$ value was obtained in nanoemulsion E5 $(-34.25 \mathrm{mV})$.

The RIs for EEO nanoemulsions (E1-E5) were obtained as $1.335-1.341$ (Table 4 ). The maximum RI value was obtained in EEO nanoemulsion E5 (1.341 \pm 0.10$)$. However, the minimum RI value was obtained in EEO nanoemulsion $\mathrm{E} 1(1.335 \pm 0.04)$

The \% T of EEO nanoemulsions (E1-E5) was obtained as 91.1-99.1\% (Table 4). EEO nanoemulsion E1 showed the maximum \% T value $(99.1 \pm 0.2 \%)$. However, EEO nanoemulsion E5 showed the minimum\% $\mathrm{T}$ value $(91.1 \pm$ $0.8 \%)$.

The TEM images of optimized formulation E1 were taken and interpreted for surface morphology and droplet diameter(Fig. 3).

\subsection{Wound healing evaluation}

The results of effect of oral administration of pure EEO and optimized nanoemulsion E1 as compared with standard gentamycin on rats are shown in Table 5 and Fig. 4.

Table 3 Qualitative results of physical stability and self-nanoemulsification tests.

\begin{tabular}{ccccc}
\hline \multirow{2}{*}{ Code } & *Self-nanoemulsification & \multicolumn{3}{c}{ Thermodynamic stability tests } \\
\cline { 2 - 5 } & test grade & Cent. & H\&T & FPT \\
\hline E1 & A & $\sqrt{ }$ & $\sqrt{ }$ & $\sqrt{ }$ \\
E2 & A & $\sqrt{ }$ & $\sqrt{ }$ \\
E3 & A & $\sqrt{ }$ & $\sqrt{ }$ \\
E4 & B & $\sqrt{ }$ & $\sqrt{ }$ & $\sqrt{ }$ \\
E5 & B & $\sqrt{ }$ & $\sqrt{ }$ \\
\hline
\end{tabular}

$\sqrt{ }$ (Passed the particular test), cent. (centrifugation), H\&T (heating and cooling cycles), FPT (freeze-pump-thaw cycles), * (all the formulations passed this test with grade $\mathrm{A}$ or $\mathrm{B}$ in the presence of water, $0.1 \mathrm{~N} \mathrm{HCl}$ and phosphate buffer)

Table 4 Different physicochemical parameters of EEO nanoemulsions (E1-E5).

\begin{tabular}{crcccc}
\hline \multirow{2}{*}{ Code } & \multicolumn{5}{c}{ Physicochemical parameters } \\
\cline { 2 - 6 } & $\Delta_{\mathrm{dm}} \pm \mathrm{SD}(\mathrm{nm})$ & PDI & $\mathrm{ZP}(\mathrm{mV})$ & $\mathrm{RI} \pm \mathrm{SD}$ & $\% \mathrm{~T} \pm \mathrm{SD}$ \\
\hline E1 & $32.45 \pm 2.84$ & 0.153 & -34.25 & $1.335 \pm 0.04$ & $99.1 \pm 0.2$ \\
E2 & $46.24 \pm 4.28$ & 0.174 & -36.21 & $1.336 \pm 0.06$ & $98.6 \pm 0.5$ \\
E3 & $61.52 \pm 5.84$ & 0.202 & -37.32 & $1.337 \pm 0.07$ & $97.8 \pm 0.6$ \\
E4 & $124.32 \pm 8.74$ & 0.242 & -38.01 & $1.340 \pm 0.09$ & $92.4 \pm 0.7$ \\
E5 & $142.35 \pm 10.58$ & 0.278 & -38.25 & $1.341 \pm 0.10$ & $91.1 \pm 0.8$ \\
\hline
\end{tabular}

Mean droplet diameter $\left(\Delta_{\mathrm{dm}}\right)$, polydispersity index (PDI), \% transmittance $(\% \mathrm{~T})$, zeta potential $(\mathrm{ZP})$, refractive index $(\mathrm{RI})$, standard deviation (SD) 


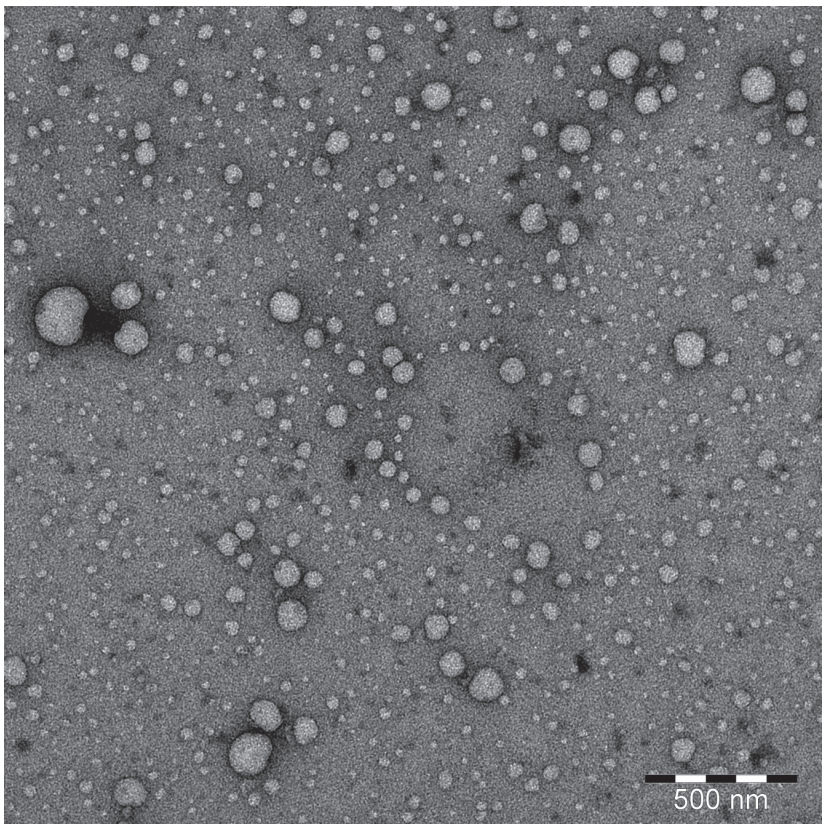

Fig. 3 TEM images of optimized EEO nanoemulsion(E1) with spherical shaped droplets.

In the research study, the rats were anaesthetized using diethyl ether due to its major advantages over other anaesthetic agents. The \% wound contraction was found to be enhanced till day 24 in both test samples (pure EEO and nanoemulsion E1) and standard gentamycin treated rats. Pure EEO and optimized nanoemulsion E1 facilitated wound contraction significantly from day 12 to 24 as compared with control $(p<0.05)$. The difference in wound contraction between optimized nanoemulsion E1 and standard was not statistically significant $(p>0.05)$. However, the wound healing abilities of optimized nanoemulsion E1 and standard were statistically significant as compared with pure $\operatorname{EEO}(p<0.05)$. The results of effect of oral administration of tests (pure EEO and nanoemulsion E1) as compared with standard on epithelization period are furnished in Table 6. The time for the complete epithelization was obtained as $13.40 \pm 0.79,12.40 \pm 0.82,9.00 \pm 0.56$ and $7.80 \pm 0.48$ days for control, pure EEO, optimized nanoemulsion E1 and standard, respectively. Epithelization time was significantly lower in standard and optimized nanoemulsion E1 treated rats as compared with pure EEO and control group rats $(p<0.05)$. However, epithelization period was not significant in optimized nanoemulsion E1 and standard $(p>0.05)$. These results suggested the potential of nanoemulsion for greater wound healing effects upon oral administration as compared with pure EEO.

\subsection{Collagen content determination}

Leucine content in terms of $\mathrm{mg} / \mathrm{g}$ of tissue in the granulation tissues of the animals on day 1 and day 10 after oral administration of pure EEO, optimized nanoemulsion E1

Table 5 Effect of oral administration of pure EEO and an optimized nanoemulsion E1 in comparison with standard gentamycin on circular excision wound model in rats at different days of treatment.

\begin{tabular}{lccccccc}
\hline \multirow{2}{*}{ Formulations } & \multicolumn{7}{c}{ Wound area of contraction \pm SEM $(\%)$} \\
\cline { 2 - 8 } & 0 Day & $4^{\text {th }}$ Day & $8^{\text {th }}$ Day & $12^{\text {th }}$ Day & $16^{\text {th }}$ Day & $20^{\text {th }}$ Day & $24^{\text {th }}$ Day \\
\hline Control & $0.00 \pm 0.00$ & $16.14 \pm 2.32$ & $30.54 \pm 2.26$ & $41.87 \pm 1.74$ & $61.18 \pm 1.23$ & $80.24 \pm 0.68$ & $98.72 \pm 0.16$ \\
Pure EEO & $0.00 \pm 0.00$ & $17.82 \pm 2.34$ & $34.51 \pm 2.58$ & $48.28 \pm 1.47$ & $67.72 \pm 1.16$ & $84.31 \pm 0.12$ & $99.84 \pm 0.09$ \\
E1 & $0.00 \pm 0.00$ & $42.88 \pm 0.72$ & $54.10 \pm 0.40$ & $75.15 \pm 0.81$ & $88.29 \pm 0.56$ & $99.49 \pm 0.16$ & $100.00 \pm 0.00$ \\
Gentamycin & $0.00 \pm 0.00$ & $61.03 \pm 0.73$ & $78.58 \pm 0.43$ & $90.25 \pm 0.67$ & $95.46 \pm 0.73$ & $99.53 \pm 0.14$ & $100.00 \pm 0.00$ \\
\hline
\end{tabular}

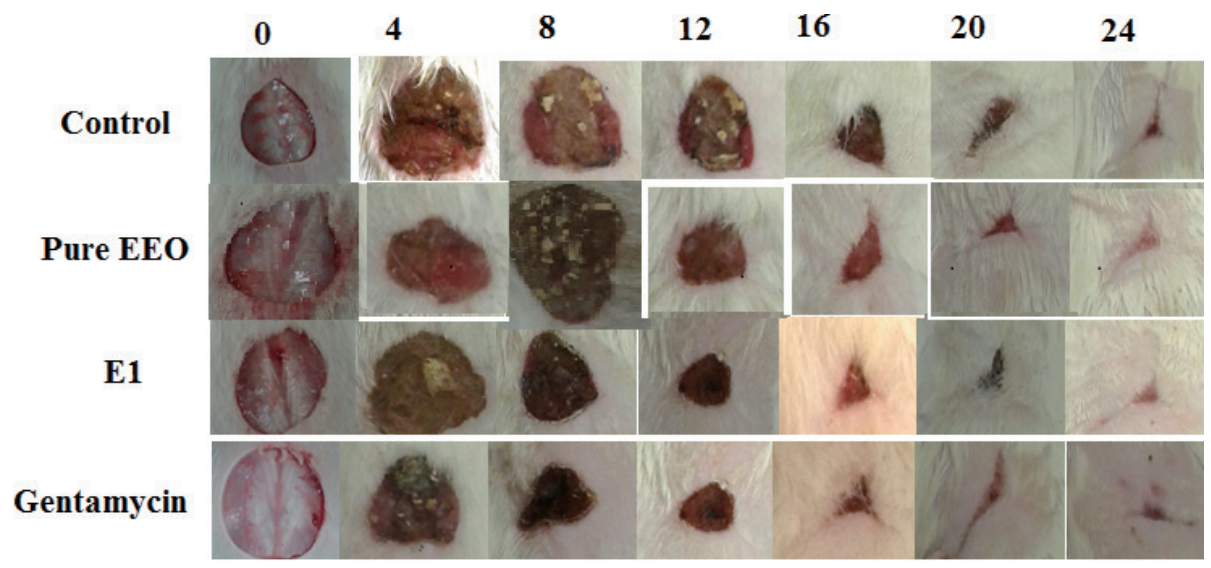

Fig. 4 Wound healing effects of pure EEO, optimized annoemulsion(E1) and gentamycin as compared with control after 0, 4, $8,12,16,20$ and 24 days of inducing wound in rats. 
Table 6 Effect of oral administration of pure EEO and an optimized nanoemulsion E1 in comparison with standard gentamycin on wound epithelization period in excision wound model in rats.

\begin{tabular}{cc}
\hline Formulations & $\begin{array}{c}\text { Period of epithelization } \pm \\
\text { SEM (Days) }\end{array}$ \\
\hline Control & $13.40 \pm 0.79$ \\
Pure EEO & $12.40 \pm 0.82$ \\
E1 & $9.00 \pm 0.56$ \\
Gentamycin & $7.80 \pm 0.48$ \\
\hline
\end{tabular}

and standard are furnished in Table 7. The leucine levels of nanoemulsion $\mathrm{E} 1$ and standard antibiotic treated rats were found to be enhanced significantly on day 10 as compared with pure EEO treated rats and negative control $(p<0.05)$.

\subsection{Histopathology of healed tissues}

The photomicrographs of histopathological examination of control, tests (pure EEO and nanoemulsion E1) and standard antibiotic treated rats are shown in Fig. 5. Histopathological examination of skin at day 12 with H\&E suggested the sign of "ulceration, edema, epithelization, granulation and abundance of mononuclear cells infiltration" in control group rats (Fig. 5a). However, the photomicrographs of pure EEO treated animals showing the sign of "less ulceration, edema and large amount of granulation as well as sign of healed skin structure with well-formed, near to normal epidermis, restoration of adnexa, and extensive fibrosis and collagen tissue" within the dermis (Fig. 5b). On the other hand, optimized nanoemulsion(E1) treated animals showed large amount of granulation tissue, small number of mononuclear inflammatory cells, and restoration of adnexa and extensive fibrosis and no sign of ulceration and edema (Fig. 5c). The animals treated with standard showed healed skin structures with well-formed, near to normal epidermis, restoration of adnexa, and extensive fibrosis and collagen tissue within the dermis (Fig. 5d). The results of histopathological examinations are furnished in

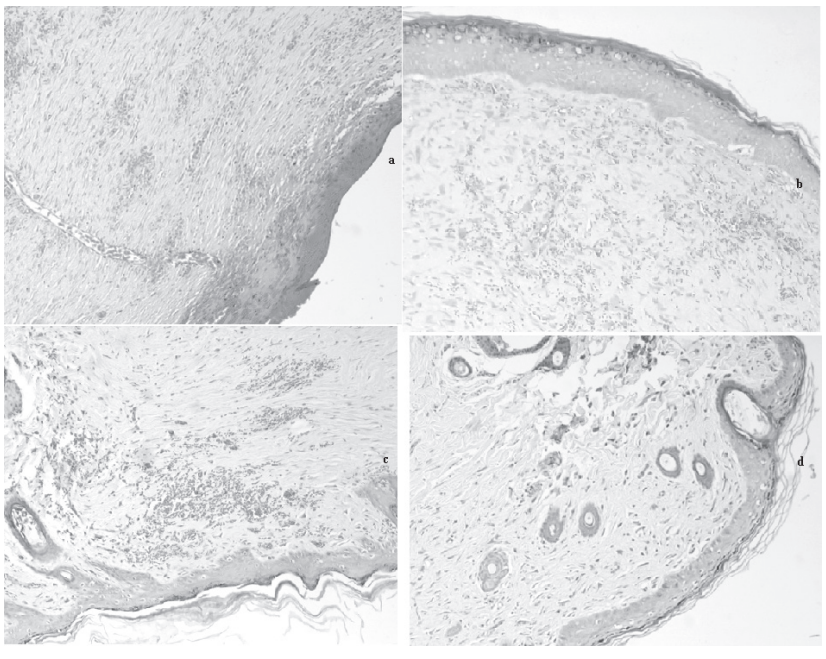

Fig. 5 Histopathology of rat skin at day 12 stained with H\&E (100x); (a) control group rats; (b) pure EEO treated rats; (c) EEO nanoemulsion (E1) treated rats; (d) gentamycin treated rats.

Table 8.

\section{DISCUSSION}

The results of aqueous phase titration suggested that the maximum zones of nanoemulsion were represented by $\mathrm{S}_{\text {mix }}$ ratio of 1:1 (Fig. 2C) and hence various nanoemulsios for EEO were selected from Fig. 2c. With respect to internal phase concentration (EEO), the entire zones in phase diagram were considered in Fig. 2c. In selected nanoemulsions (E1-E5), the concentration of EEO was varied from $12-28 \% \mathrm{w} / \mathrm{w}$ and the concentration of $\mathrm{S}_{\text {mix }}$ was kept constant at $24 \% \mathrm{w} / \mathrm{w}$. It was observed that all nanoemulsions were physically stable at three different steps of these tests. The presence of cosurfactant(Transcutol)along with surfactant(Tween-85) in nanoemulsions might be the main reason for physical stability of nanoemulsions ${ }^{28)}$. The surfactant alone is not capable to reduce interfacial tension between oil phase and aqueous phase up to the required value $^{29)}$. However, the presence of cosurfactant along with

Table 7 Estimation of collagen content after oral administration of pure EEO and an optimized nanoemulsion E1 in comparison with standard gentamycin on circular excision wound model in rats at $1^{\text {st }}$ and $10^{\text {th }}$ day of treatment.

\begin{tabular}{cccc}
\hline \multicolumn{4}{c}{ Collagen \pm SEM $(\mathrm{mg}$ of leucine/g of tissue) } \\
\hline Formulations & $1^{\text {st }}$ day & $10^{\text {th }}$ day & Negative control \\
\hline Pure EEO & $0.22 \pm 0.07$ & $0.50 \pm 0.03$ & $0.33 \pm 0.04$ \\
E1 & $0.25 \pm 0.05$ & $0.65 \pm 0.02$ & $0.33 \pm 0.04$ \\
Gentamycin & $0.28 \pm 0.03$ & $0.98 \pm 0.03$ & $0.33 \pm 0.04$ \\
\hline
\end{tabular}


Table 8 The median histopathologic scores for wound healing effects determined after oral administration of pure EEO and an optimized nanoemulsion E1 in comparison with and control and standard gentamycin by using a modified 0 to 5 numerical scale; the scores were 0 for absence, 1 for occasional presence, 2 for light scattering, 3 for abundance, 4 for confluence of cells and 5 for fibres.

\begin{tabular}{cccccc}
\hline Formulations & Epithelialisation & $\begin{array}{c}\text { Inflammatory } \\
\text { cell infiltration }\end{array}$ & $\begin{array}{c}\text { Fibroblast } \\
\text { proliferation }\end{array}$ & Neovascularization & $\begin{array}{c}\text { Collagen } \\
\text { deposition }\end{array}$ \\
\hline Gentamycin & $5.22 \pm 0.34$ & $0.89 \pm 0.51$ & $5.40 \pm 0.38$ & $4.10 \pm 0.23$ & $5.27 \pm 0.61$ \\
E1 & $3.80 \pm 0.52$ & $1.71 \pm 0.35$ & $3.58 \pm 0.67$ & $2.34 \pm 0.56$ & $3.64 \pm 0.57$ \\
Pure EEO & $2.48 \pm 0.41$ & $3.67 \pm 0.64$ & $2.53 \pm 0.71$ & $1.45 \pm 0.34$ & $2.24 \pm 0.54$ \\
Control & $0.88 \pm 0.43$ & $5.52 \pm 0.27$ & $0.72 \pm 0.01$ & $0.92 \pm 0.31$ & $1.05 \pm 0.36$ \\
\hline
\end{tabular}

surfactant could reduce the interfacial tension up to negative value and systems become highly stable ${ }^{28-30)}$. The objective of self-nanoemulsification test was to evaluate any precipitation/phase separation upon mild agitation or dilution with "water, acid buffer $(0.1 \mathrm{~N} \mathrm{HCl})$ and phosphate buffer ( $\mathrm{pH} 6.8) " 16,30$ ). The results of this test suggested that EEO was maintained in solubilized form at molecular level in developed nanoemulsions and its self-nanoemulsification behavior was independent of $\mathrm{pH}^{35)}$. The droplet diameter of nanoemulsions was found to be decreased significantly with decrease in the concentration of EEO in formulation. The results suggested that EEO/oil phase had significant impact on droplet diameter of EEO nanoemulsions. The largest droplet diameter was obtained in formulation E5 $(142.35 \pm 10.58 \mathrm{~nm})$ that was due to the presence of highest concentration of $\mathrm{EEO}(28 \% \mathrm{w} / \mathrm{w})$ in formulation $\mathrm{E} 5$. However, the droplet diameter of EEO nanoemulsion E1 was obtained as lowest $(32.45 \pm 2.84 \mathrm{~nm})$ that was due to the presence of lowest concentration of $\operatorname{EEO}(12 \% \mathrm{w} / \mathrm{w})$ in formulation E1. The highest PDI value was obtained in EEO nanoemulsion E5 (0.278). Overall, the PDIs were recorded as $<0.3$ in all nanoemulsions, suggesting uniformity of droplet diameter in all nanoemulsions. The determination of ZP is useful in evaluation of surface charge and stability of prepared nanoemulsions. The results of ZP determination suggested stable formation of EEO nanoemulsions. The determination of RI is helpful in evaluation of transparent nature and nanoemulsion type. The RIs values of all nanoemulsions were much closed with that of water (1.333), suggesting transparent nature and oil-in-water type behavior of all EEO nanoemulsions. The determination of $\% \mathrm{~T}$ is also helpful in evaluation of transparent nature of nanoemulsions. The results of $\% \mathrm{~T}$ suggested transparent behavior of all EEO nanoemulsions. TEM analysis was conducted for the evaluation of the surface morphology of optimized EEO nanoemulsion E1. The size of all internal droplets of formulation E1 was obtained within nanometer range as shown in Fig. 3. The shape of nanoemulsion droplets was recorded as spherical. The enhanced wound healing effects of optimized nanoemulsion E1 were possible due to nanosized droplet dimeter of nanoemulsion and the presence of solubilizers including Tween-85 and Transcutol in optimized nanoemulsion E1. The potential of producing wound contraction by EEO and EEO loaded nanoemulsion obtained in this study suggested that Eucalyptus plant possesses a definite prohealing action because most of the wound healing occurred due to wound contraction $^{36)}$. The wound healing effects of pure EEO and EEO nanoemulsion could be due to definite enhancement in the proliferation of epithelial cells as proposed in literature ${ }^{37}$.

Many essential oils have been studied for their wound healing effects on different animal models ${ }^{38-41)}$. Nanoemulsions are known to enhance oral absorption and therapeutic efficacy of drugs ${ }^{11-15)}$ and essential oils have potential for wound healing effects ${ }^{38-41)}$. Therefore, nanoemulsions of EEO were prepared in order to enhance wound healing effects of EEO in this study. It was proposed that the restoration and the functional integrity of the wound tissue involve various processes including "inflammation, wound contraction, angiogenesis, extracellular matrix deposition and tissue remodeling". Either single or multiple mechanisms could be responsible in different phases of wound healing which can contribute to the overall outcome of the wound healing process ${ }^{42,43)}$. At day 4 post-wounding, a significant reduction in swelling and exudates in rats treated with an optimized nanoemulsion E1 was recorded. These effects were comparable to standard antibiotic treated rats and higher than control and pure EEO as shown in Fig. 4.

It has been proposed that the internal oil phase/lipoidal portion of nanoemulsions enhance the intestinal lymphatic uptake of lipophilic compounds which could further help in avoidance of pre-systemic metabolism of these compounds ${ }^{44)}$. This potential of nanoemulsions could definitely results in rapid absorption of drugs from nanoemulsions and finally results in enhanced oral bioavailability and therapeutic efficacy of such lipophilic compounds ${ }^{44,45)}$. Hence, the enhanced wound healing effects of EEO nanoemulsion $\mathrm{E} 1$ were possibly due to enhancement in lymphatic uptake of EEO from nanoemulsions and avoidance of pre-systemic metabolism. In the present study, leucine assay was used for collagen determination in wound tissues ${ }^{46,47)}$. The collagen accumulation is the sum of synthesis and destruction 
which occur simultaneously during wound healing process $^{48)}$. Therefore, the enhanced levels of leucine in optimized nanoemulsion E1 and standard antibiotic treated rats suggested enhanced collagen content in these sample matrices. However, the level of leucine was much lower in case of negative control which was possible be due to a prolonged inflammatory phase in negative control. Overall, the formulation of EEO in terms of nanoemulsion was advantageous in terms of collagen content because the level of collagen in rats treated with optimized nanoemulsion E1 was much higher as compared with pure EEO and negative control. The results of histopathological evaluation suggested that the wound healing and repair are accelerated by EEO in the form of nanoemulsion.

\section{CONCLUSIONS}

Different nanoemulsion formulations of EEO were prepared and investigated for its wound healing potential in this study. Nanoemulsios of EEO were prepared using aqueous phase titration method and characterized for various physicochemical parameters. Based on various physicochemical parameters including minimum droplet diameter, least PDI, optimal ZP value, optimal RI value and maximum\% $\mathrm{T}$ value, nanoemulsion formulation $\mathrm{E} 1 \mathrm{was}$ optimized and selected for wound healing activity in rats. Wound healing potential of optimized nanoemulsion was found to be significant as compared with pure EEO and control. However, it was comparable with standard antibiotic. Optimized nanoemulsion formulation also showed significant enhancement in the level of collagen as compared with pure EEO and negative control. Histopathological examinations of optimized nanoemulsion treated rats showed no signs of inflammatory cells which suggested that prepared nanoemulsion was safe and nontoxic to animals. The results of this study suggested the potential of nanoemulsion for oral delivery of EEO for enhancing its wound healing potential.

\section{CONFLICT OF INTEREST}

"The authors declare that they have no conflict of interest with this manuscript."

\section{ACKNOWLEDGEMENT}

“This project was financially supported by King Saud University, Vice Deanship of Research Chairs, Kayyali Chair for Pharmaceutical industry through the grant number FN2017."

\section{References}

1) Miguel, M.G. Antioxidant and anti-inflammatory activities of essential oils: A short review. Molecules 15, 9252-9287 (2010).

2) Bakkali, F.; Averbeck, S.; Averbeck, D.; Idaomar, M. Biological effects of essential oils-a review. Food Chem. Toxicol. 46, 446-475 (2008).

3) Bos, R.; Kayser, O.; Quax, W.J. Essential oil constituents of Piper cubeba L. fils. from Indonesia. J. Essent. Oil Res. 19, 14-17(2007).

4) Adorjan, B. Biological properties of essential oils: an updated review. Flavour Fragr. J. 1, 407-426 (2010).

5) Silva, J.; Abebe, W.; Sousa, S.M.; Duarte, V.G.; Machado, M.I.L.; Matos, F.J.A. Analgesic and anti-inflammatory effects of essential oils of Eucalyptus. J. Ethnopharmacol. 89, 277-283(2003).

6) Sebei, K.; Sakouhi, F.; Herchi, W.; Khouja, M.L.; Boukhchina, S. Chemical composition and antibacterial activities of seven Eucalyptus species essential oil leaves. Biol. Res. 48, E7 (2015).

7) Siramon, P.; Ohtani, Y. Antioxidative and antiradical activities of Eucalyptus camaldulensis leaf oils from Thailand. J. Wood Sci. 53, 498-504(2007).

8) Su, Y.C.; Ho, C.L.; Wang, E.I.; Chang, S.T. Antifungal activities and chemical compositions of essential oils from leaves of four eucalyptus. Taiwan J. For. Sci. 21, 49-61(2006).

9) Cimanga, K.; Kambu, K.; Tona, L.; Apers, S.; De Bruyne, T.; Hermans, N. et al. Correlation between chemical composition and antibacterial activity of essential oils of some aromatic medicinal plants growing in the Democratic Republic of Congo. J. Ethnopharmacol. 79, 213-220 (2002).

10) Goodger, J.Q.D.; Woodrow, I.E. Selection gains for essential oil traits using micropropagation of Eucalyptus polybractea. For. Ecol. Manag. 255, 3652-3658 (2008).

11) Shakeel, F.; Shazly, G.A.; Raish, M.; Ahmad, A.; Kalam, M.A.; Ali, N. et al. Biological investigation of supersaturated self-nanoemulsifying drug delivery system of Piper cubeba essential oil. RSC Adv. 5, 105206105217 (2015).

12) Alam, P.; Ansari, M.J.; Anwer, M.K.; Raish, M.; Kamal, Y.K.T.; Shakeel, F. Wound healing effects of nanoemulsion containing clove essential oil. Art. Cell Nanomed. Biotechnol. 45, 591-597(2017).

13) Jain, S.; Kambam, S.; Thanki, K.; Jain, A.K. Cyclosporine A loaded self-nanoemulsifying drug delivery system(SNEDDS): implication of a functional excipient based co-encapsulation strategy on oral bioavailability and nephrotoxicity. RSC Adv. 5, 49633-49642 (2015).

14) Sharma, G.; Beg, S.; Thanki, K.; Katare, O.P.; Jain, S.; Kohli, K. et al. Systematic development of novel cationic self-nanoemulsifying drug delivery systems of 
candesartan cilexetil with enhanced biopharmaceutical performance. $R S C A d v$. 5, 71500-71513(2015).

15) Villar, A.M.; Naveros, B.C.; Campmany, A.C.; Trenchs, M.A.; Rocabert, C.B.; Bellowa, L.H. Design and optimization of self-nanoemulsifying drug delivery systems (SNEDDS) for enhanced dissolution of gemfibrozil. Int. J. Pharm. 431, 161-175 (2012).

16) Shakeel, F.; Haq, N.; El-Badry, M.; Alanazi, F.K.; Alsarra, I.A. Ultra fine super self-nanoemulsifying drug delivery system (SNEDDS) enhanced solubility and dissolution of indomethacin. J. Mol. Liq. 180, 89-94 (2013).

17) Gebrehiwot, M.; Asres, K.; Bisrat, D.; Mazumder, A.; Lindemann, P.; Bucar, F. Evaluation of the wound healing property of Commiphora guidottii Chiov. ex. Guid. BMC Compl. Alt. Med. 15, E282 (2015).

18) Farhapour, M.R.; Habibi, M. Evaluation of the wound healing activity of an ethanolic extract of Ceylon cinnamon in mice. Vet. Med. 2012, 53-57 (2012).

19) Cavalcanti, J.M.; Leal-Cardoso, J.H.; Diniz, L.R.L.; Portella, V.G.; Costa, C.O.; Linard, C.F. et al. The essential oil of Croton zehntneri and trans-anethole improves cutaneous wound healing. J. Ethnopharmacol. 144, 240-247 (2012).

20) Tumen, I.; Suntar, I.; Keles, H.; Akkol, E.K. A therapeutic approach for wound healing by using essential oils of Cupressus and Juniperus species growing in Turkey. Evi. Based Compl. Alt. Med. 2012, E788281 (2012).

21) Suntar, I.; Akkol, E.K.; Tosun, A.; Keles, H. Comparative pharmacological and phytochemical investigation on the wound-healing effects of the frequently used essential oils. J. Essent. Oil Res. 26, 41-49 (2014).

22) Tumen, I.; Suntar, I.; Eller, F.J.; Keles, H.; Akkol, E.K. Topical wound-healing effects and phytochemical composition of heartwood essential oils of Juniperus virginiana L., Juniperus occidentalis Hook., and Juniperus ashei J. Buchholz. J. Med. Food 16, 48-55 (2013).

23) Umasankar, K.; Nambikkairaj, B.; Backyavathi, M.D. Wound healing activity of topical Mentha piperita and Cymbopogan citratus essential oil on streptozotocin induced rats. Asian J. Pharm. Clin. Res. 6, 180-183 (2013).

24) Dwivedi, V.; Tripathi, S. Review study on potential activity of Piper betle. J. Pharmacog. Phytochem. 3, 93-98 (2014).

25) Wong, C.M.; Ling, J.J. In vitro study of wound healing potential in black pepper (Piper nigrum L.). UK. J. Pharm. Biosci. 2, 5-9(2014).

26) Ouoba, M.A.; Koudou, J.; Some, N.; Ouedraogo, S.; Guissou, I.P. Wound healing and antibacterial properties of leaf essential oil of Vitex simplicifolia oliv. from Burkina Faso. in Chromatography and its ap- plications (Dhanarasu, S. ed.). ISBN: 978-953-510357-8(2012).

27) Suqumar, S.; Ghosh, V.; Nirmala, M.J.; Mukherjee, A.; Chandrasekaran, N. Ultrasonic emulsification of eucalyptus oil nanoemulsion: antibacterial activity against Staphylococcus aureus and wound healing activity in Wistar rats. Ultarason. Sonochem. 21, 1044-1049 (2014).

28) Baboota, S.; Shakeel, F.; Ahuja, A.; Ali, J.; Shaiq, S. Design, development and evaluation of novel nanoemulsion formulations for transdermal potential of celecoxib. Acta Pharm. 57, 315-332 (2007).

29) Shakeel, F.; Baboota, S.; Ahuja, A.; Ali, J.; Aqil, M.; Shaiq, S. Nanoemulsions as vehicles for transdermal delivery of aceclofenac. AAPS PharmSciTech. 8, E104 (2007).

30) Shaiq, S.; Shakeel, F.; Talegaonkar, S.; Ahmad, F.J.; Khar, R.K.; Ali, M. Development and bioavailability assessment of ramipril nanoemulsion formulation. Eur. J. Pharm. Biopharm. 66, 227-243(2007).

31) Dash, G.K.; Suresh, P.; Ganapathy, S. Studies on hypoglycemic and wound healing activities of Lantana camara Linn. J. Nat. Remed. 1, 105-110 (2002).

32) Werner, S.; Breeden, M.; Hubner, G.; Greenhalgh, D.G.; Longaker, M.T. Induction of keratinocyte growth factor expression is reduced and delayed during wound healing in the genetically diabetic mouse. J. Invest. Dermatol. 103, 469-473(1994).

33) Moore, S.; Stein, W.H. Photometric ninhydrin method for use in the chromatography of amino acids. J. Biol. Chem. 176, 367-388 (1948).

34） Mandl, I.; MacLennan, J.D.; Howes, E.L.; DeBellis, R.H.; Sohler, A. Isolation and characterization of proteinase and collagenase CL. Histolyticum. J. Clin. Invest. 32, 1323-1329 (1953).

35) Shakeel, F.; Haq, N.; Alanazi, F.K.; Alsarra, I.A. Surface-adsorbed reverse micelle-loaded solid self-nanoemulsifying drug delivery system of talinolol. Pharm. Dev. Technol. 21, 131-139(2016).

36) Ejaz, S.; Chekarova, I.; Cho, J.W.; Lee, S.Y.; Ashraf, S.; Lim, S.W. Effect of aged garlic extract on wound healing: a new frontier in wound management. Drug Chem. Toxicol. 32, 191-203(2009).

37) Getie, M.; Gebre-Mariam, T.; Rietz, R.; Hohne, C.; Huschka, C.; Schmidtke, M. Evaluation of the antimicrobial and anti-inflammatory activities of the medicinal plants Dodonaea viscosa, Rumex nervosus and Rumex abyssinicus. Fitoterapia 74, 139-143 (2003).

38) Kumar, B.; Vijayakumar, M.; Govindarajan, R.; Pushpangadan, P. Ethnopharmacological approaches to wound healing-exploring medicinal plants of India. $J$. Ethnopharmacol. 114, 103-113(2007).

39) de Fatima, A.; Modolo, L.V.; Sanches, A.C.; Porto, R.R. Wound healing agents: the role of natural and non-nat- 
ural products in drug development. Mini Rev. Med. Chem. 8, 879-888(2008).

40) Tumen, I.; Akkol, E.K.; Suntar, I.; Keles, H. Wound repair and anti-inflammatory potential of essential oils from cones of Pinaceae: preclinical experimental research in animal models. J. Ethnopharmacol. 137, 1215-1220 (2011).

41) Suntar, I.; Akkol, E.K.; Keles, H.; Oktem, A.; Baser, K.H.; Yesilada, E. A novel wound healing ointment: a formulation of Hypericum perforatum oil and sage and oregano essential oils based on traditional Turkish knowledge. J. Ethnopharmacol. 134, 89-96 (2011).

42) Velnar, T.; Bailey, T.; Smrkolj, V. The wound healing process: An overview of the cellular and molecular mechanisms. J. Int. Med. Res. 37, 1528-1542 (2009).

43) Grieb, G.; Steffens, G.; Pallua, N.; Bernhagen, J.; Bucala, R. Circulating fibrocytes-biology and mechanisms in wound healing and scar formation. Int. Rev. Cell Mol. Biol. 291, 1-19 (2011).

44) Kalepu, S.; Manthina, M.; Padavala, V. Oil-based drug delivery systems-a review. Acta Pharm. Sin. B. 3, 361-372 (2013).

45) Sureshkumar, R.; Gowthamarajan, K.; Bhavani, P. Nanoemulsion for lymphatic absorption: investigation of fenofibrate nanoemulsion system for lymphatic uptake. Int. J. Chem. Tech. Res. 7, 832-841(2015).

46) Nayak, S.; Nalabothu, P.; Sandiford, S.; Bhogadi, V.; Adogwa, A. Evaluation of wound healing activity of $A l-$ lamanda cathartica L. and Laurus nobilis L. extracts on rats. BMC Complem. Altern. Med. 6, E12 (2006).

47) Taşkiran, D.; Taşkiran, E.; Yercan, H.; Kutay, F.Z. Quantification of total collagen in rabbit tendon by the Sirius Red method. Turkish J. Med. Sci. 29, 7-9 (1999).

48) Iyyam Pillai, S.; Palsamy, P.; Subramanian, S.; Kandaswamy, M. Wound healing properties of Indian propolis studied on excision wound-induced rats. Pharm. Biol. 48, 1198-1206(2010). 